\title{
Tagungsbericht: Lokalrundfunktage 2019 in Nürnberg
}

Audio erlebt in den letzten Monaten einen regelrechten Boom. Ausgelöst wurde er in erster Linie durch Musikstreaming-Dienste, Podcasts und Smartspeaker. Was bedeutet das für die Radiobranche? Bei den 27. Lokalrundfunktagen, die am 2. und 3. Juli 2019 in Nürnberg stattgefunden haben, drehte sich alles um Innovationen, neue Technologien und Produktentwicklungen, durch die auch lokale Sender vom Audioboom profitieren können.

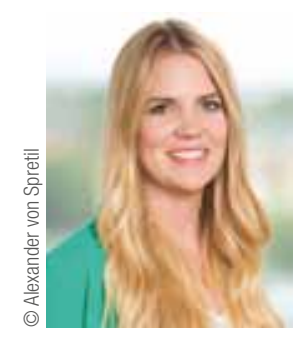

Kerstin Grünewald

Medientage München

PR \& Marketing

kerstin.gruenewald@medientage.de

Schlüsselbegriffe: Audioboom I Lokal-TV I Lokal-Radio I Podcast I Innovation

Siegfried Schneider, Präsident der Bayerischen Landeszentrale für neue Medien, riet den lokalen Anbietern einerseits „mit Mut in die digitale Zukunft zu gehen“, andererseits

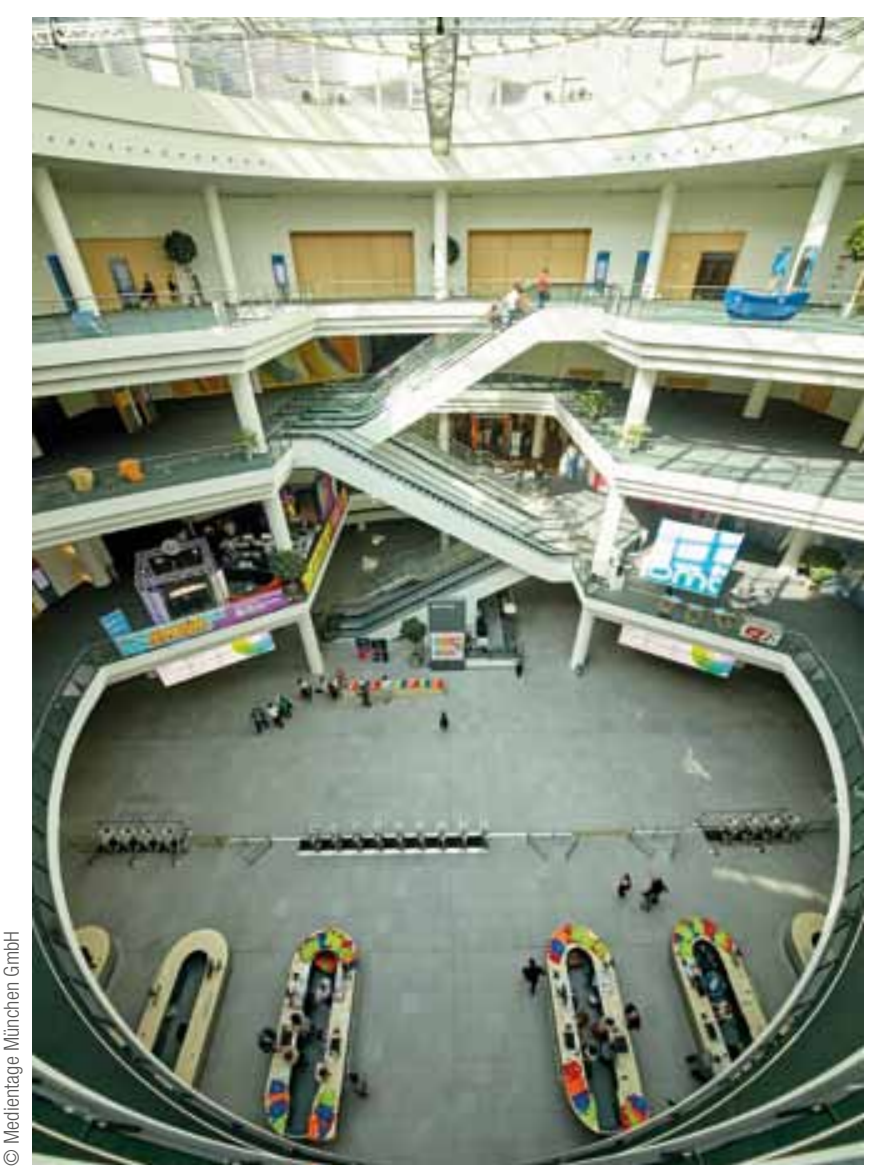

aber auch Initiative zu zeigen, damit die bayerische private Radio- und Fernsehlandschaft die bundesweit vielfältigste und erfolgreichste bleibe. Die wichtigsten Aufgaben für die lokalen Sender sind laut Schneider gute lokale Inhalte, Präsenz auf allen digitalen Kanälen und mehr Kooperationen untereinander.

„Radio ist spontan. Radio ist emotional. Radio ist lokal. Radio hat Information und Musik. Diese vielen Vorzüge des Radios, für die es Menschen lieben, muss es in der digitalen Welt mehr denn je ausspielen. Der Mut zur lokalen Marke, der direkte Draht zu den Menschen vor Ort ist in der globalen digitalen Welt das Pfund, mit dem es zu wuchern gilt: Nachrichten aus dem eigenen Umfeld gibt es nur vor Ort", so Schneider.

Wie es Moderatoren schaffen, eine Sendung unvergesslich zu machen und wie man sein Publikum wirklich gewinnen kann, zeigte David Lloyd, Programmberater aus Großbritannien. In seiner Keynote „Effective Radio Presentation“ sprach er über die Wirkung von Sprache auf die Zuhörer: Je näher die eigene Art zu sprechen und das, worüber man spricht, an der Zielgruppe sei, desto besser erreiche man die Hörerinnen und Hörer. Geschichten aus der Du-Perspektive würden sie mehr einbeziehen, wodurch sowohl die Verweildauer als auch die Interaktion höher werde: „Wer

Bei den 27. Lokalrundfunktagen drehte sich 2019 alles um Innovationen, neue Technologien und Produktentwicklungen, durch die lokale Sender vom Audioboom profitieren können. 


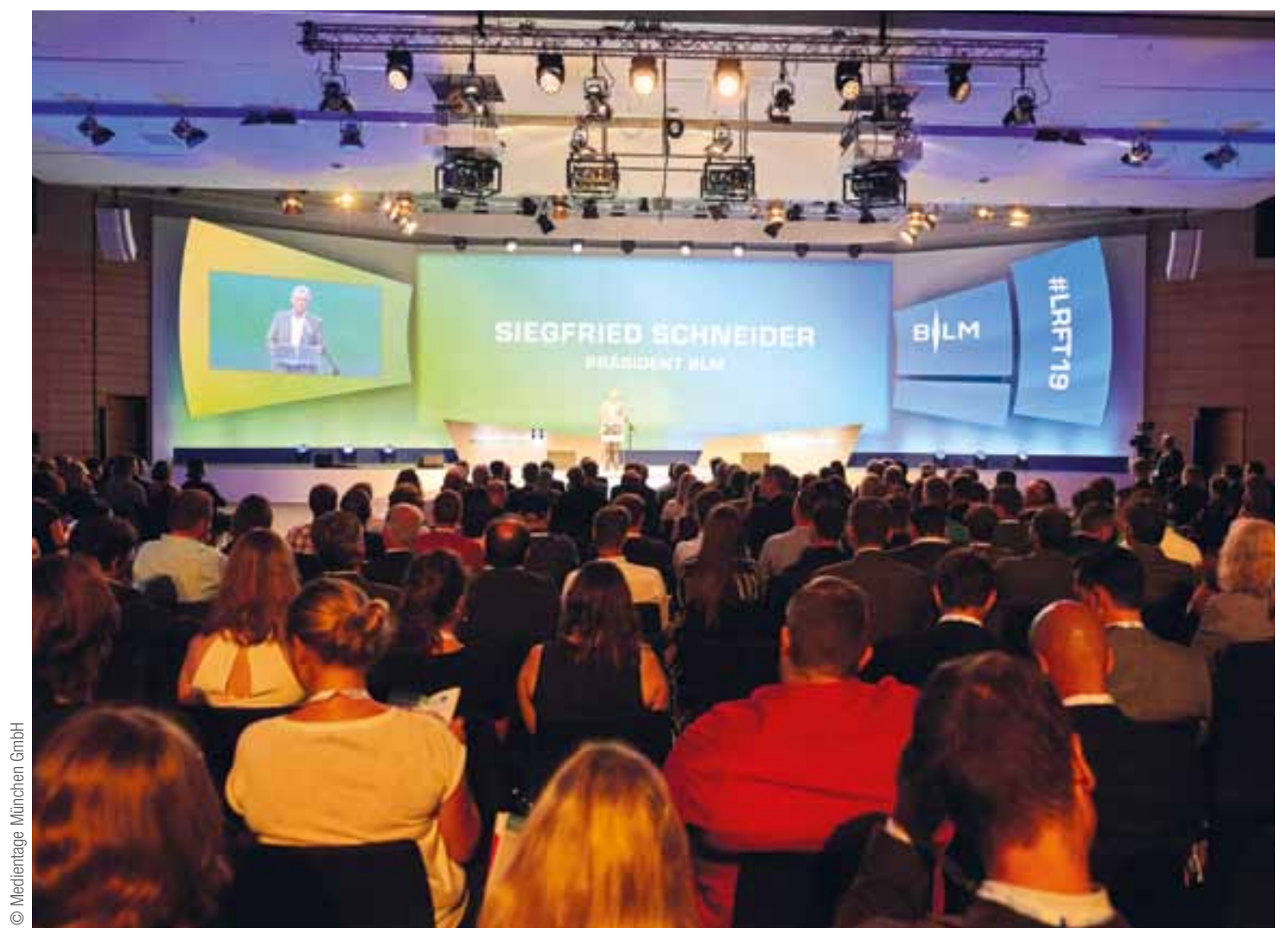

Siegfried Schneider, Präsident der Bayerischen Landeszentrale für neue Medien, riet den lokalen Anbietern "mit Mut in die digitale Zukunft zu gehen“.

sein Publikum wirklich versteht, bekommt die besten Reaktionen".

Erfolgreiche Unterhaltungsformate, neues Storytelling im Lokal-TV, genauso wie neue Technologien und Verbreitungswege wurden auch mit Blick auf das Fernsehen thematisiert. Eine Live-Schalte gab es etwa zur Show „Dorfheldentour" von TV Mainfranken nach Kürnach, wo die Bewohner einen Platz zum Weltraum umgestalten mussten. Fazit: Jede Stadt und jede Region bieten außergewöhnliche Orte und damit auch Geschichten - die Redakteure müssen sie nur entdecken.

Erstmals gab es bei den Lokalrundfunktagen auch ein Podcast-Special. Neben Podcast-Formaten von und für Radiosender und Podcast-Advertising-Beispielen wurde gezeigt, wie auch lokale und regionale Radioanbieter den Audio-Boom für sich nutzen können. Rob Szymoniak, ehemaliger Radiomann, jetzt Gründer und Geschäftsführer der Podcast-Full-Service-Agentur Podcastmania, zeigte, wie mit einfachen Mitteln gehaltvoller Content produziert werden kann und wie somit neue Zielgruppen erreicht werden können. „Je nerdiger, spitzer und spezieller das Thema, desto höher die Attraktion für die Hörer», ist Szymoniak überzeugt. Zudem hätten besonders Radiomacher dank in- rer technischen sowie inhaltlichen Expertise das Potenzial, spannende Geschichten zu erzählen.

Neben Vorträgen und Diskussionen mit über 70 nationalen und internationalen Referenten gab es aber auch einige Gründe zu feiern: Die BLM-Hörfunk- und Lokalfernsehpreise 2019 wurden im Rahmen der Lokalrundfunktage verliehen. „Die Gewinner erzählen aufwändige und spannende Geschichten mit tollen Protagonisten - und das immer tagesaktuell und lokal“, so der Jury-Vorsitzende Dr. Torsten Rossmann. In diesem Jahr wurden die Preise in 12 verschiedenen Kategorien vergeben. 


\section{Kongresse - Tagungen - Events}

Oktober 2019

08.10.2019-09.10.2019

IFRA WORLD PUBLISHING EXPO /

DCX DIGITAL CONTENT EXPO

BERLIN

„Next Business for Publishers and Content Strategists"

16.10.2019-20.10.2019

FRANKFURTER BUCHMESSE

FRANKFURT AM MAIN

Ausstellungs- und Messe GmbH des Börsenvereins des

Deutschen Buchhandels

22.10.2019

TAG DER DIGITALISIERUNG

HAMBURG

HafenCity Universität Hamburg

23.10.2019 - 25.10.2019

33. MEDIENTAGE MÜNCHEN

MÜNCHEN

„Next Digital Level: Let's Build the Media we want!”

ICM/Messe München

24.10.2019: PANEL „Forum Medienmanagement" unserer Fachzeitschrift MedienWirtschaft in Kooperation mit dem Bayerischen Rundfunk

Thema: „Müssen Medien Haltung zeigen? Verantwortung in der digitalen Welt"

November 2019

04.11.2019

VDZ PUBLISHERS‘ SUMMIT

BERLIN

Kongress der Zeitschriftenverleger

05.11.2019

BDZV-VERTRIEBSGIPGEL

KÖLN

ZV Akademie

06.11.2019-08.11.2019

DGPUK FACHGRUPPE DIGITALE KOMMUNIKATION

BERLIN

JAHRESTAGUNG

Thema: „Automating Communication in the Networked

Society: Contexts, Consequences, Critique"

In Kooperation mit dem Weizenbaum-Institut für die

vernetzte Gesellschaft
08.11.2019-10.11.2019

BUCH BASEL

BASEL

Internationales Literatur Festival

13.11.2019

HIGHTECH SUMMIT BADEN-WÜRTTEMBERG

EUROPAPARK RUST

Tagungskongress der Initiative Wirtschaft 4.0

18.11.2019 - 19.11.2019

22. MEDIENFORUM MITTWEIDA

MITTWEIDA

Hochschule Mittweida

20.11.2019 - 21.11.2019

DEUTSCHER HANDELSKONGRESS

BERLIN

Jahreskongress für den deutschen Einzelhandel und seine Partner

Dezember 2019

04.12.2019-05.12.2019

46. DEUTSCHER MARKETING TAG DÜSSELDORF

„Europas führende Konferenz im Bereich Marketing“ Deutscher Marketing Verband / Management Forum der Handelsblatt Media Group GmbH

Januar 2020

07.01.2020 - 10.01.2020

CES Las Vegas - CONSUMER ELECTRONIC SHOW LAS VEGAS

29.01.2020 - 30.01.2020

DEUTSCHER MEDIENKONGRESS 2020

FRANKFURT AM MAIN

Alte Oper Frankfurt

Februar 2020

24.02.2020 - 27.02.2020

MWC - GSMA MOBILE WORLD CONGRESS 2020

BARCELONA

Leitmesse der Mobilfunkbranche 


\author{
10.03.2020 - 11.03.2020 \\ INTERNET WORLD EXPO 2020 - \\ The Commerce E-Experience \\ MÜNCHEN
}

Ebner Verlag GmbH \& Co. KG Büro München

\subsubsection{0 - 15.03.2020 \\ LEIPZIGER BUCHMESSE 2020 \\ LEIPZIG}

Leipziger Messe GmbH

24.03.2020 - 25.03.2020

DIGITAL INNOVATORS‘ SUMMIT 2020

BERLIN

FIPP, VDZ Verband Deutscher Zeitschriftenverleger e.V.

24.03.2020 - 28.03.2020

DIDACTA

STUTTGART

Die Bildungsmesse

Didacta Verband / Verband Bildungsmedien

April 2020

01.04.2020 - 02.04.2020

BIG-DATA.AI SUMMIT 2020 - BITKOM

BERLIN

hub.berlin

18.04.2020 - 22.04.2020

NAB SHOW - National Association of Broadcasters

(NAB)

LAS VEGAS

$N A B$, die weltgrößte Messe für elektronische Medien

23.04.2020 - 24.04.2020

SGKM-JAHRESTAGUNG 2020

WINTERTHUR/SCHWEIZ

"Media Literacy"”

Mai 2020

13.05.2020 - 14.05.2020

MEDIENTAGE MITTELDEUTSCHLAND 2020

LEIPZIG

AG MTM e. V. c/o Medienanstalt Sachsen-Anhalt

\section{Ihr Portal}

\section{für Medien \& Recht}

- Titelarchiv mit über 70.000 geschützten Titeln

- aktuelle Titel-Anmeldungen

- frische News über Urteile, Kanzleien, Gesetze und Prozesse

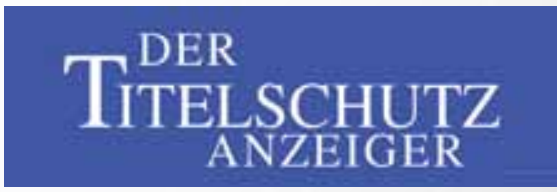

Weitere Informationen bei Birgit Weselmann Tel 040 609009-57

titelschutz-anzeiger@titelschutzanzeiger.de www.titelschutzanzeiger.de 